\title{
Minimal prognostic significance of sentinel lymph node metastasis in patients with CT1-2 and cN0 breast cancer
}

\author{
Hideo Shigematsu ${ }^{1 *}$ D, Mai Nishina ${ }^{1}$, Daisuke Yasui ${ }^{1}$, Taizo Hirata ${ }^{2}$ and Shinji Ozaki ${ }^{1}$
}

\begin{abstract}
Background: The prognostic value of sentinel lymph node (SLN) metastases may be minimized by the limited disease burden of lymph node metastases and tailoring adjuvant therapy based on breast cancer biology. The aim of this study is to assess the prognostic significance of SLN metastasis in patients with CT1-2NOMO breast cancer.

Patients and methods: Between January 2006 and December 2015, 582 patients underwent SLN biopsy for CT1-2NOMO breast cancers. CNO was essentially diagnosed by ultrasound sonography. The prognostic values of SLN metastases were retrospectively evaluated.

Results: Among 582 patients with CT1-2NOMO breast cancer, 111 patients (19.1\%) were positive for SLN metastasis, including 39 cases (6.7\%) of micrometastasis and 72 cases (12.4\%) of macrometastases. The median size of SLN metastasis was $3.0 \mathrm{~mm}$ (range $0.2-16 \mathrm{~mm}$, mean $4.1 \mathrm{~mm}$ ). In log-rank test, presence of SLN metastasis was not associated with breast cancer recurrence $(p=0.21)$; 5 -year and 10-year recurrence-free survival (RFS) were 93.0\% and $96.5 \%$, and $93.0 \%$ and $90.4 \%$ in the SLN-positive and SLN-negative groups, respectively. In the propensity score matching cohort $(n=178)$, there was no significant difference in RFS between the SLN-positive and SLN-negative groups $(p=0.90)$. In Cox regression analysis, a continuous value of Ki67 expression was a significant prognostic factor (HR 1.03; 95\% Cl 1.01-1.05, $p=0.017$ ).

Conclusion: SLN metastasis has a minimal impact on RFS for patients with cT1-2NOMO breast cancer in the modern medical era. A proliferation marker is a better factor for poor prognosis than the presence of SLN metastases in this population.
\end{abstract}

Keywords: Breast cancer, SLN, Minimal, Prognosis, Proliferation marker

\section{Introduction}

Sentinel lymph node (SLN) biopsy is a standard procedure for assessing axillary lymph node metastasis in a patient with clinically node-negative (cN0) breast cancer $[1,2]$. Although the therapeutic value of axillary surgery is denied in patients with clinical T1-T2N0 (cT1-2N0) breast cancer [2,3], pathological nodal status is still an important poor prognostic factor for decisions regarding adjuvant therapy. In a patient with SLN metastasis, adjuvant chemotherapy and radiotherapy are administered in cases with clinically node-positive $(\mathrm{cN}+)$ breast cancer.

\footnotetext{
* Correspondence: shigematu1330@yahoo.co.jp

${ }^{1}$ Department of Breast Surgery, National Hospital Organization Kure Medical Center and Chugoku Cancer Center, 3-1, Aoyama-cho, Kure City, Hiroshima 737-0023, Japan

Full list of author information is available at the end of the article
}

However, the prognostic value of SLN metastasis in patients with cT1-2N0M0 breast cancer has become controversial with limited disease burden in SLN due to the advances in preoperative axillary evaluation and the introduction of modern tailored therapy based on breast biology [4]. A previous report showed that axillary ultrasound sonography (AUS) for patients with cT1-2 breast cancer had a sensitivity of $70 \%$ and a negative predictive value of $84 \%$ for detection of lymph node involvement [5]. Meta-analysis of AUS-guided needle cytology or biopsy of axillary lymph nodes in patients with invasive breast cancer estimated $79.6 \%$ of sensitivity, $98.3 \%$ of specificity, and 97.1\% of positive predictive value for axillary staging [6]. AUS-guided cytology or biopsy of suspicious lymph node was also shown to accurately exclude clinically significant

(C) The Author(s). 2019 Open Access This article is distributed under the terms of the Creative Commons Attribution 4.0 International License (http://creativecommons.org/licenses/by/4.0/), which permits unrestricted use, distribution, and 
lymph node metastasis [7, 8]. Patients with cN0 breast cancer diagnosed by AUS are expected to have a limited disease burden of the axilla, which raises the hypothesis that the presence of SLN metastasis has the minimal prognostic value. Although the anatomical stage of breast cancer staging still has clinical significance, breast cancer biology has become the most important prognostic and predictive factor for determining adjuvant therapy [9]. Tailoring adjuvant therapy based on breast cancer biology has resulted in the significant improvement of prognosis in early-stage breast cancer $[10,11]$. Considering advances in preoperative axillary evaluation and tailored therapy based on breast biology, the prognostic value of SLN metastasis in cNO breast cancer should be reevaluated.

The aim of this study is to assess the prognostic value of SLN metastases in patients with cT1-2N0M0 breast cancer in the modern medical era. We retrospectively evaluated the prognostic impact of SLN metastases in this population.

\section{Materials and methods Patients and methods}

A total of 582 consecutive patients with clinically T1-2N0M0 invasive breast cancers underwent sentinel lymph node biopsy (SLNB) for axillary staging at the Kure Medical Center and the Chugoku Cancer Center, Kure, Japan, between January 2006 and December 2015. This study retrospectively evaluated the prognostic value of SLN metastasis. The Kure Medical Center review board approved this study (30-05). The requirement for informed consent from individual patients was waived because this was a retrospective review of a prospectively maintained patient database.

\section{Clinicopathological factors}

The clinicopathological factors in this retrospective study retrieved from our prospectively maintained database included age at surgery, T factor, SLN status, estrogen receptor (ER) status, progesterone receptor (PgR) status, human epidermal growth factor receptor 2 (HER2) status, nuclear grade, Ki67 index, and prescriptions of adjuvant chemotherapy/radiotherapy. ER and PgR status were evaluated by immunohistochemical (IHC) assays, and $\geq 1 \%$ positively stained tumor cells were classified as positive. HER2 status was evaluated by IHC and fluorescence in situ hybridization (FISH) individually or in combination. HER2-positive tumors were defined as those with an IHC score of 3+ or those showing HER2 gene amplification using FISH, in accordance with the ASCO guidelines [12].

Adjuvant systemic and/or radiation therapy was administered as clinically indicated [9]. Patients with hormone receptor (HR)-positive breast cancers were treated with adjuvant endocrine therapy for at least
5 years. Administration of adjuvant chemotherapy for luminal-type breast cancer was determined based on the risk for breast cancer recurrence and preference. Patients with HR-negative breast cancer were administered adjuvant chemotherapy. Patients with HER2-positive breast cancer were administered adjuvant trastuzumab concomitant with taxane-based chemotherapy and subsequent trastuzumab therapy for 1 year in total. Patients were followed in accordance with clinical guidelines that included clinical examination, annual mammography, and additional imaging tests in cases with signs of recurrence. Recurrencefree survival (RFS) was defined as the elapsed time from the date of surgery until the date of the first event (relapse or death from any cause) or of last follow-up.

\section{Evaluation of clinical node-negative and sentinel lymph node procedure}

Clinical axillary nodal status was essentially evaluated by ultrasound sonography. Abnormal findings of axillary lymph nodes included the following: cortical thickness, abnormal morphologic characteristics, or loss of fatty hilum. Lymph nodes with abnormal findings were subsequently examined by fine needle aspiration cytology or core needle biopsy for the diagnosis of metastasis. Patients with negative findings from AUS and/or pathological evaluations by AUS-guided biopsy were diagnosed as having $\mathrm{CNO}$. SLNB was performed to provide a final pathological diagnosis.

The SNLB procedure has been described in a previous report [13]. Briefly, SLNB is performed using both a dye colloid and radioisotopes. SLNB is performed at the same time along with the primary breast tumor resection. Sampled SLNs are evaluated for metastases using both the one-step nucleic acid amplification (OSNA) assay and histological evaluation. In the OSNA assay, OSNA- $\left(\right.$ CK19 mRNA $<2.5 \times 10^{2}$ copies $\left./ \mu \mathrm{L}\right)$ is diagnosed as negative, and OSNA+ $\left(2.5 \times 10^{2}\right.$ to $<5.0 \times 10^{3}$ copies $/ \mu \mathrm{L})$ and OSNA++ $\left(\geq 5.0 \times 10^{3}\right.$ copies $\left./ \mu \mathrm{L}\right)$ are diagnosed as positive for SLN metastases. In histological evaluation, no or isolated $(<0.2 \mathrm{~mm})$ tumor cells is diagnosed as negative, and micrometastases $(0.2-2 \mathrm{~mm})$ or macrometastases $(<2 \mathrm{~mm})$ is recognized as positive for SLN metastases. In OSNA assay, OSNA- is diagnosed as negative, and OSNA+ and OSNA++ are regarded as micrometastases and macrometastases, respectively.

During this study period, axillary lymph node dissections were essentially performed in cases with SLN-metastases. $\mathrm{N}$ stage was determined by the number of lymph node metastasis according to tumor, node, metastasis (TNM) staging classification for breast cancer.

\section{Statistical analysis}

The association between clinicopathological factors and SLN status was assessed using the $\chi 2$ test. Kaplan-Meier 
survival curves and the log-rank test were used to determine the univariate significance of the variables. A Cox regression model was used to examine multiple covariates for survival.

Because the presence of SLN metastasis is thought to affect decisions regarding adjuvant chemotherapy or may be affected by tumor size or biological factors, a propensity score analysis was applied to control confounding factors. The propensity score matching included the following factors: age at surgery $(<55$ vs. $\geq 55)$, $\mathrm{T}$ stage (T1 vs. T2), estrogen receptor status (positive vs. negative), progesterone receptor status (positive vs. negative), HER2 status (positive vs. negative), nuclear grade (1, 2 vs. 3 ), Ki67 index (<14\% vs. $\geq 14 \%)$, adjuvant chemotherapy (yes vs. no), and adjuvant radiation therapy (yes vs. no). Pairs of patients were identified using the propensity scores; one patient with SLN negative was randomly matched with a SLN positive, using the nearest matching neighbor within a caliper. The caliper coefficient was determined as 0.01 . After adjustment with propensity score matching, differences in clinicopathology between groups were compared using the chi-square test.

A $p$ value of $<0.05$ was considered as statistically significant. Statistical analyses were done using JMP statistics software version 13.2.1 for Windows (SAS Institute, Inc.).

\section{Results}

Clinicopathological factors

Of the 582 patients with cT1-2N0 breast cancer, 471 patients $(81 \%)$ were negative for SLN metastasis and 111 patients (19.1\%) were positive for SLN metastasis, including 100 (17\%) N1 and 10 (2\%) N2 disease. Among 111 cNOSLN + cases, the final pathological examination revealed 39 cases $(6.7 \%)$ of micrometastasis and 72 cases (12.4\%) of macrometastases. The median size of SLN metastasis was $3.0 \mathrm{~mm}$ (range $0.2-16 \mathrm{~mm}$, mean 4.1 $\mathrm{mm})$. Table 1 shows clinicopathological factors according to SLN status. Between the two groups, the probabilities of a T2 tumor $(p<0.0001)$ and the administration of adjuvant chemotherapy $(p<0.0001)$ were significantly higher in the SLN-positive group compared with the SLN-negative group. Otherwise, there was no significant difference in regard to age, ER, PgR, HER2, nuclear grade, Ki67 index, and adjuvant radiotherapy. Clinicopathological factors after propensity score matching are shown in Table 1.

\section{Prognostic value of SLN metastasis in CT1-2NO breast cancer}

Within the median follow-up period from definitive surgery for primary breast cancer of 4.9 years, 27 patients (4.6\%) had breast cancer recurrences. In univariate

Table 1 Clinicopathological factors according to the status of SLN in cNO breast cancer before and after propensity score matching

\begin{tabular}{|c|c|c|c|c|c|c|c|c|c|c|c|}
\hline \multirow[t]{3}{*}{ Factor } & & \multicolumn{5}{|c|}{ All patients $(n=582)$} & \multicolumn{5}{|c|}{ Matched patients $(n=178)$} \\
\hline & & \multicolumn{2}{|c|}{ CNOSN- $(n=471)$} & \multicolumn{2}{|c|}{$\mathrm{CNOSN}+(n=111)$} & \multirow[b]{2}{*}{$p$ value } & \multicolumn{2}{|c|}{ CNOSN- $(n=89)$} & \multicolumn{2}{|c|}{ cNOSN+ $(n=89)$} & \multirow[b]{2}{*}{$p$ value } \\
\hline & & $N$ & $\%$ & $N$ & $\%$ & & N & $\%$ & $N$ & $\%$ & \\
\hline \multirow[t]{2}{*}{ Age } & $\geq 55$ & 150 & 31.8 & 36 & 32.4 & 0.91 & 34 & 38.2 & 29 & 32.6 & 0.43 \\
\hline & $<55$ & 321 & 68.2 & 75 & 67.5 & & 55 & 61.8 & 60 & 67.4 & \\
\hline \multirow[t]{2}{*}{ T factor } & $\mathrm{T} 1$ & 354 & 75.2 & 57 & 51.4 & $<0.0001$ & 51 & 57.3 & 51 & 57.3 & 1 \\
\hline & $\mathrm{T} 2$ & 117 & 24.8 & 54 & 48.6 & & 38 & 42.7 & 38 & 42.7 & \\
\hline \multirow[t]{2}{*}{ ER } & Positive & 367 & 77.9 & 92 & 82.9 & 0.24 & 77 & 86.5 & 77 & 86.5 & 1 \\
\hline & Negative & 104 & 22.1 & 19 & 17.1 & & 12 & 13.5 & 12 & 13.5 & \\
\hline \multirow[t]{2}{*}{$\mathrm{PgR}$} & Positive & 320 & 67.9 & 83 & 74.8 & 0.15 & 64 & 71.9 & 65 & 73 & 0.87 \\
\hline & Negative & 151 & 22.1 & 28 & 25.2 & & 25 & 28.1 & 24 & 27 & \\
\hline \multirow[t]{2}{*}{ HER2 } & Negative & 390 & 82.8 & 93 & 83.8 & 0.8 & 79 & 88.8 & 79 & 88.8 & 1 \\
\hline & Positive & 81 & 17.2 & 18 & 16.2 & & 10 & 11.2 & 10 & 11.2 & \\
\hline \multirow[t]{2}{*}{ Nuclear grade } & 1,2 & 345 & 73.3 & 84 & 75.7 & 0.6 & 69 & 67.5 & 69 & 67.5 & 1 \\
\hline & 3 & 126 & 26.7 & 27 & 24.3 & & 20 & 22.5 & 20 & 22.5 & \\
\hline \multirow[t]{2}{*}{ Ki67 } & $\geq 14 \%$ & 170 & 36.1 & 31 & 27.9 & 0.11 & 22 & 24.7 & 22 & 24.7 & 1 \\
\hline & $<14 \%$ & 293 & 63.9 & 77 & 72.1 & & 67 & 75.3 & 67 & 75.3 & \\
\hline \multirow[t]{2}{*}{ Adjuvant chemotherapy } & Yes & 153 & 32.5 & 66 & 59.5 & $<0.0001$ & 49 & 55.1 & 49 & 55.1 & 1 \\
\hline & No & 318 & 67.5 & 45 & 40.5 & & 40 & 44.9 & 40 & 44.9 & \\
\hline \multirow[t]{2}{*}{ Adjuvant radiotherapy } & Yes & 308 & 65.4 & 69 & 62.2 & 0.52 & 56 & 62.9 & 57 & 64 & 0.88 \\
\hline & No & 163 & 34.6 & 42 & 37.8 & & 33 & 37.1 & 32 & 36 & \\
\hline
\end{tabular}

ER estrogen receptor, $P g R$ progesterone receptor, HER2 human epidermal receptor 2 
analysis with a log-rank test, the SLN status was not a significant prognostic factor $(p=0.21)$ (Fig. 1a). The 5-year and 10-year RFS were 93.0\%, 96.5\%, and 93.0\%, 90.4\% in the SLN-positive and SLN-negative group, respectively. In regard to the size of metastases, the 5-year and 10 -year RFS were $94.0 \%, 91.0 \%$, and $94.0 \%, 91.0 \%$ in SLN micrometastases and SLN macrometastases, respectively (log-rank test, $p=0.57$ ) (Additional file 1 : Figure S1). In the propensity score matching cohort $(n=178)$, there was no significant difference in RFS between a SLN-positive and SLN-negative group ( $p=0.90)$ (Fig. 1b). On the other hand, nuclear grade $3(p=0.013)$, a higher Ki67 index $(p=0.007)$, and T2 $(p=0.004)$ were significantly associated with worse RFS in a log-rank test (Fig. 2). There was a tendency for a worse prognosis in ER-negative $(p=0.11)$ breast cancer. Cox regression analysis showed that a continuous value of Ki67 expression was a significant prognostic factor (HR 1.03; 95\% CI, $1.01-1.05, p=0.017)$. A higher Ki67 index $(>14 \%)$ was a marginal significant poor prognostic factor for RFS (HR 3.16, 95\% confidential interval 0.99-14.02, $p=0.051$ ). On the other hand, the presence of SLN metastasis was not a significant prognostic factor $(p=0.44)$. In exploratory analysis, the prognoses of patients with $\mathrm{cT} 1-2 \mathrm{cN}+\mathrm{M} 0$ breast cancer were compared with patients with cT1-2cN0M0 breast cancer, and there was a significant difference in RFS between these groups. The 5 -year and 10 -year RFS were $92.0 \%, 70.0 \%$, and $92.0 \%, 68.0 \%$ in the cNOSLN-positive and cN-positive group, respectively $(p<0.0001, \log$-rank test) (Additional file 2: Figure S2). $\mathrm{cN}$ positive was still a significant poor prognostic factor in Cox regression analysis (HR 4.4, 95\% CI 1.8-11.8, $p=0.0006)$ (Table 2).

\section{Discussion}

In this study, we showed that the presence of SLN metastases has a minimal impact on prognosis in patients with cT1-2N0M0 breast cancer in the modern medical era. The 5-year and 10-year RFS were 93.0\%, 96.5\%, and 93.0\%, 90.4\% in a SLN-positive and SLN-negative group, respectively, and there was no significant difference in RFS between these groups ( $p=0.2$, log-rank test). After propensity score matching between the cNOSLN+ group and the cNOSLN- group, there was still no significant difference in RFS. On the other hand, a proliferation marker is a better factor for poor prognosis than the presence of SLN metastases in this population.

The presence of axillary lymph node metastasis has been regarded as an important prognostic factor in patients with non-metastatic breast cancer $[14,15]$; however, the prognostic value is considered to be influenced by tumor burden in involved lymph nodes in patients who underwent SLN biopsy for cNO breast cancer. Isolated tumor cell (ITC) (not greater than $0.2 \mathrm{~mm}$ ) in SLN is regarded as pathological node negative (pN0) because the presence of ITC does not confer a deteriorated prognosis. The clinical significance of micrometastases $(>0.2 \mathrm{~mm}$ but $<2.0 \mathrm{~mm})$ in breast cancer remains controversial; prospective observational analyses by Andersson et al. showed poor prognostic significance for micrometastases in SLN [16, 17]; however, data from a NSABP B32 trial, ACOSOG Z0010 trial, and MD Anderson Cancer Center showed minimal impact from micrometastases on survival outcome in patients who underwent SLN biopsy $[18,19]$. The presence of macrometastases in SLNs $(>2.0 \mathrm{~mm})$ is conventionally regarded as an established poor prognostic factor; however, the prognostic value of
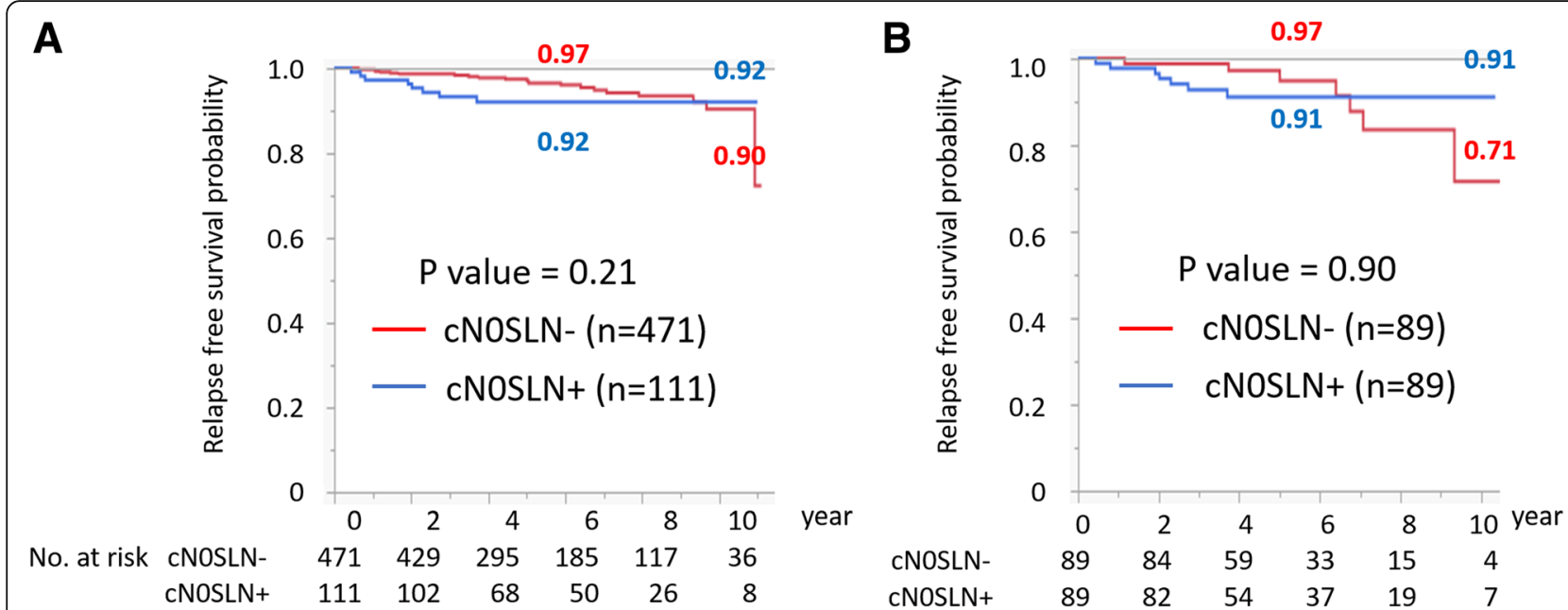

Fig. 1 Prognostic value of SLN metastasis in CT1-2NOMO breast cancer. Relapse-free survival a of all patients $(n=582)$ stratified by the presence of SLN metastasis and $\mathbf{b}$ of patients stratified by the presence of SLN metastasis among propensity score-matched patients $(n=178)$. $p$ value was evaluated using the log-rank test. cNO clinical node negative, SLN sentinel lymph node 

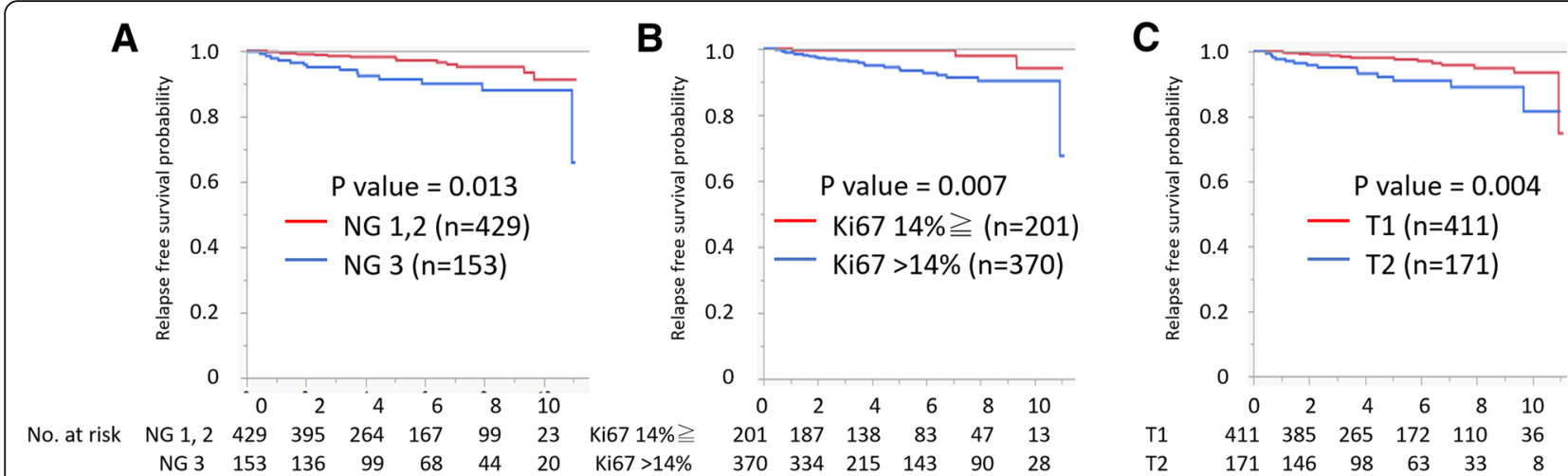

Fig. 2 Prognostic value of Ki67 index and T stage in cT1-2N0M0 breast cancer. Relapse-free survival stratified by a nuclear grade, $\mathbf{b}$ Ki67 index, and $\mathbf{c} T$ stage. $p$ value was evaluated using the log-rank test

macrometastases in SLN may be decreasing in the modern medical era. A previous report of 647 patients with cT1-2N0 breast cancer by Tucker et al., in which cN0 was diagnosed by AUS, patients with false-negative results of AUS had an equivalent RFS to patients with a pathologic node-negative disease [5]. Our study also evaluated cNO by AUS and/or AUS-guided biopsy and showed no significant difference in RFS between a SLN-positive and SLN-negative group in patients with cT1-2N0M0 breast cancer. In this study, because the proportion of adjuvant chemotherapy is significantly affected by the presence of SLN metastases, propensity score analysis was applied to control confounding factors. After the propensity score matching, there was still no significant difference in RFS between the cNOSLN + and cNOSLN- group. On the other hand, $\mathrm{cN}+$ is still a significant prognostic factor in cT1-2 breast cancer. The prognosis of patients with $\mathrm{cT} 1-2$ and $\mathrm{cN}+$ breast cancer

Table 2 Multivariate analyses of predictors for distant diseasefree survival and overall survival $(n=582)$

\begin{tabular}{llll}
\hline Factor & HR & $(95 \%$ Cl) & $p$ value \\
\hline Age ( 55 vs. > 55) & 1.3 & $(0.55-3.33)$ & 0.56 \\
T factor (T2 vs. T1) & 1.56 & $(0.66-3.64)$ & 0.3 \\
SLN factor (SLN+ vs. SLN-) & 1.42 & $(0.56-3.36)$ & 0.44 \\
ER (positive vs. negative) & 0.81 & $(0.26-2.7)$ & 0.73 \\
PgR (positive vs. negative) & 1.21 & $(0.42-3.82)$ & 0.73 \\
HER2 (positive vs. negative) & 0.64 & $(0.20-1.69)$ & 0.38 \\
Nuclear grade (3 vs. 1, 2) & 1.39 & $(0.53-3.64)$ & 0.51 \\
Ki67 & & & \\
$\quad$ Ki67 (<14\% vs. $\geq 14 \%)$ & 3.16 & $(0.99-14.02)$ & 0.051 \\
$\quad$ Ki67 (continuous value) & 1.03 & $(1.01-1.05)$ & 0.017 \\
Adjuvant chemotherapy (yes vs. no) & 1.2 & $(0.48-3.09)$ & 0.69 \\
Adjuvant radiotherapy (yes vs. no) & 0.71 & $(0.31-1.69)$ & 0.29 \\
\hline
\end{tabular}

$E R$ estrogen receptor, $P g R$ progesterone receptor, HER2 human epidermal receptor $2, S L N$ sentinel lymph node was compared with those with cT1-2cN0 and SLN+ breast cancer, and the 5-year and 10-year RFS were $92.0 \%$, $70.0 \%$, and $92.0 \%, 68.0 \%$ in the cNOSLN+ and cN+SLN group, respectively. The previous report by Tucker et al. also showed that the RFS for patients with $\mathrm{cN}+$ disease was significantly worse than for patients with $\mathrm{cNO}$ and $\mathrm{SLN}+$ disease. These findings suggest that clinically detectable lymph node metastasis has a clinically meaningful tumor burden, which has a significant survival impact for patients with breast cancer.

The decreased impact of SLN metastasis on breast cancer survival can be explained by advances in axillary imaging evaluation and adjuvant systemic therapy. First, AUS-based axillary evaluation prior to axillary surgery can exclude a significant tumor burden in lymph nodes. In this study, cNO diagnosed by AUS or AUS-guided needle biopsy showed a false-negative rate of $19.1 \%$, including $6.7 \%$ of micrometastasis and $12.4 \%$ of macrometastases. This result is similar with the previous report by Mittendorf et al. [19]; the false-negative rate was $22.6 \%$, including $8.8 \%$ of micrometastasis and $13.8 \%$ of macrometastases. In contrast, a previous report by Andersson et al. showed a higher probability of false negatives (25.8\%) and macrometastases (22.2\%), in which the presence of SLN metastases was evaluated as a significant factor for poor prognosis. The tumor burden of false-negative lymph nodes may affect the prognostic significance of SLN metastases. In regard to the accuracy of AUS for detection of axillary disease, recent studies have shown consistent and sufficient results. Cyr et al. reported the ability of AUS to exclude clinically significant metastases in the axillary node, and the NPV of AUS for identification of macrometastases was $96.9 \%$ in cT1-2N0 breast cancer [4]. Nakamura et al. reported that a proportion of patients with a significant number of lymph node metastases $(\geq 3)$ was $16 \%$ and $5 \%$ in a cN0-FNA group and cN0-CNB group, respectively [7]. These findings show that preoperative axillary evaluation 
by AUS can exclude clinically significant metastases of axillary lymph nodes in cT1-2N0M0 breast cancer. Second, presences of lymph node metastases seem to have less impact on survival in cNO breast cancer compared with biologic factors. In regard to micrometastases, retrospective analyses of phase III trial and large observational study showed no significant impact on prognosis of occult metastases in cN0 breast cancer $[18,19]$. In these analyses, endocrine responsiveness and grade were more important prognostic factors compared with micrometastases. Our study and a retrospective study by Tucker et al. [5] also showed equivalent RFS of patients with $\mathrm{cN} 0$ and pathologically node-positive disease to those with $\mathrm{cNO}$ and pNO disease; however, two thirds of node-positive patients had macrometastases in these studies. In these studies, cN0 was essentially diagnosed by AUS. In addition, the proliferation marker Ki67 index was a significant poor prognostic factor for RFS in our study. Third, during the period of this study, guidelines for adjuvant therapy have changed to emphasize the responsiveness [9]. In luminal subtype, adjuvant chemotherapy was conventionally determined by risk category defined by nodal status, $\mathrm{T}$ stage, grade, vascular invasion, and age [20]; however, recent adjuvant chemotherapy recommendation is based on the endocrine responsiveness. In luminal A-like subtype, adjuvant chemotherapy is considered in a case with four or more node involvement. In luminal B-like subtype, adjuvant chemotherapy is recommended irrespective of nodal status [21]. In HER2 subtype, adjuvant chemotherapy with HER2-targeting therapy was not indicated in a patient with a primary tumor $<1 \mathrm{~cm}$ of size and with no axillary node involvement, and recent guideline expanded the indication of adjuvant therapy to T1bN0 HER2-positive disease considering the substantial recurrence risk [22]. In TNBC, adjuvant chemotherapy is essential in both node-positive and node-negative disease. These findings suggest that tumor biology is more important for deciding adjuvant therapy and predicting recurrence than SLN metastases in patients with cT1-2N0M0 breast cancer. Finally, the therapeutic value of axillary surgery is denied in patients with clinical T1-T2N0 (cT1-2N0) breast cancer. In $\mathrm{cNO}$ breast cancer, an omission of axillary lymph node dissection (ALND) resulted in an increment of the rate of axillary failure; however, this local failure did not lead to an increment of distant metastasis or a survival disadvantage [23]. In cT1-2NOSLN + breast cancer treated with appropriate adjuvant systemic therapy and radiotherapy, ALND failed to show an improvement of local control nor long-term prognosis in spite of the possibility of residual disease in non-SLN $[2,3]$. Thus, omission of axillary surgery itself does not result in deteriorated prognosis in patients with cT1-2N0M0.
There are ongoing randomized clinical trials comparing SLN biopsy to no axillary surgery in patients with cT1-2N0M0 breast cancer, in which cN0 is evaluated by AUS and/or ultrasonography-guided biopsy [4, 24]. Because SLN biopsy is associated with surgical complications including pain, paresthesias, seroma, lymphedema, and delayed wound healing, SLN biopsy should be spared if these randomized studies show the effectiveness and safety of omission of SLN biopsy in cT1-2 N0 breast cancer. Considering the possibility of omission of axillary surgery, clinical diagnosis of $\mathrm{cNO}$ disease becomes crucially important. Although several innovative techniques, such as contra-enhanced AUS, MRI, or PET-CT, are utilized for detection of lymph node metastasis, morphological evaluation by AUS is still the standard procedure for preoperative axillary staging. Although the ability of AUS for detection of lymph node metastasis is dependent on an individual clinician's skill, the accuracy of AUS for detection of lymph node metastasis seems to be similar across studies. Indeed, the negative predictive value of $81 \%$ for detection of lymph node involvement in our study is consistent with previous reports. Considering this consistency and universality, AUS is the preferable procedure for exclusion of clinical significant lymph node metastasis.

Our study has several limitations. One limitation was the relatively short follow-up period (median, 4.9 years). However, we think that this follow-up period is enough to show the minimal prognostic impact of SLN metastasis in patients with cT1-2 and cN0 breast cancer. In luminal subtype, the annual recurrence rate is consistent up to 10 years and early recurrence is a certain surrogate marker of late recurrence. In non-luminal subtype, most recurrence occurs within 5 years and early recurrence is the primary endpoint for survival. Although the evaluation of late recurrence may confer additional survival information, the prognostic value of SLN metastasis in this study will not be altered. Second, this retrospective analysis from a single institution could have biases, and a multiple-institutional prospective study is needed to confirm our results. Finally, there was a different proportion of subtype in recurrent breast cancer between SLN+ and SLN- groups in this study. In SLN+ group, 4 out of 8 recurrent cases were non-luminal subtype, which contributed the increment of early recurrence. On the other hand, 16 out of 20 recurrent cases were luminal subtype, which contributed the increments of late recurrence. This uneven distribution of subtype in recurrent cases leaded the different relapse-free survival curves before 5 years and at 10 years in SLN-positive and SLN-negative groups.

In conclusion, our study showed that the presence of SLN metastasis has a minimal impact on prognosis in patients with cT1-2N0M0 breast cancer. A proliferation 
marker is a better factor for poor prognosis than occult nodal metastases. SLN biopsy may be spared if prospective randomized studies show the effectiveness and safety of omission of SLN biopsy in CT1-2N0M0 breast cancer.

\section{Additional files}

Additional file 1: Figure S1. Relapse-free survival stratified by the size of SLN metastasis. $p$ value was evaluated using the log-rank test. Abbreviations: CNO: clinical node negative, SLN: sentinel lymph node. (TIF $163 \mathrm{~kb}$ )

Additional file 2: Figure S2. Relapse-free survival stratified by $\mathrm{CN}$ status and SLN status. $p$ value was evaluated using the log-rank test. Abbreviations: CNO: clinical node negative, SLN: sentinel lymph node. (TIF $223 \mathrm{~kb}$ )

\section{Acknowledgements}

The authors would like to thank the Department of Diagnostic Pathology for their technical assistance.

\section{Disclaimers}

None. This has never been presented/published before in any form. All authors declare that conflicts of interest do not exist.

\section{Funding}

There was no research support for this study.

\section{Availability of data and materials}

Please contact the author for data requests.

\section{Authors' contributions}

HS helped in the conception, design, and writing of the study. MN, DY, and $\mathrm{TH}$ helped in the data curation and analysis. SO helped in the conception, design, and project administration. All authors read and approved the final manuscript.

\section{Ethics approval and consent to participate}

This study was approved by the ethics committee of the Kure Medical Center review board approved this study (30-05) in accordance with the Declaration of Helsinki. The requirement for informed consent from individual patients was waived because this was a retrospective review of a prospectively maintained patient database.

\section{Consent for publication}

Not applicable.

\section{Competing interests}

The authors declare that they have no competing interests.

\section{Publisher's Note}

Springer Nature remains neutral with regard to jurisdictional claims in published maps and institutional affiliations.

\section{Author details}

${ }^{1}$ Department of Breast Surgery, National Hospital Organization Kure Medical Center and Chugoku Cancer Center, 3-1, Aoyama-cho, Kure City, Hiroshima 737-0023, Japan. ²Department of Medical Oncology, National Hospital Organization Kure Medical Center and Chugoku Cancer Center, Kure City, Hiroshima, Japan.

Received: 5 September 2018 Accepted: 18 February 2019 Published online: 23 February 2019

\section{References}

1. Krag DN, Anderson SJ, Julian TB, Brown AM, Harlow SP, Costantino JP, Ashikaga T, Weaver DL, Mamounas EP, Jalovec LM, et al. Sentinel-lymph-node resection compared with conventional axillary-lymph-node dissection in clinically node-negative patients with breast cancer: overall survival findings from the NSABP B-32 randomised phase 3 trial. Lancet Oncol. 2010;11:927-33.
2. Giuliano AE, Ballman $K$, McCall $L$, Beitsch $P$, Whitworth PW, Blumencranz $P$, Leitch AM, Saha S, Morrow M, Hunt KK. Locoregional recurrence after sentinel lymph node dissection with or without axillary dissection in patients with sentinel lymph node metastases: long-term follow-up from the American College of Surgeons Oncology Group (Alliance) ACOSOG Z0011 randomized trial. Ann Surg. 2016;264:413-20.

3. Donker M, van Tienhoven G, Straver ME, Meijnen P, van de Velde CJ, Manse RE, Cataliotti L, Westenberg AH, Klinkenbijl JH, Orzalesi L, et al. Radiotherapy or surgery of the axilla after a positive sentinel node in breast cancer (EORTC 10981-22023 AMAROS): a randomised, multicentre, open-label, phase 3 non-inferiority trial. Lancet Oncol. 2014;15:1303-10.

4. Cyr AE, Tucker N, Ademuyiwa F, Margenthaler JA, Aft RL, Eberlein TJ, Appleton CM, Zoberi I, Thomas MA, Gao F, Gillanders WE. Successful completion of the pilot phase of a randomized controlled trial comparing sentinel lymph node biopsy to no further axillary staging in patients with clinical T1-T2 N0 breast cancer and normal axillary ultrasound. J Am Coll Surg. 2016;223:399-407.

5. Tucker NS, Cyr AE, Ademuyiwa FO, Tabchy A, George K, Sharma PK, Jin LX, Sanati S, Aft R, Gao F, et al. Axillary ultrasound accurately excludes clinically significant lymph node disease in patients with early stage breast cancer. Ann Surg. 2016;264:1098-102.

6. Houssami N, Ciatto S, Turner RM, Cody HS 3rd, Macaskill P. Preoperative ultrasound-guided needle biopsy of axillary nodes in invasive breast cancer: meta-analysis of its accuracy and utility in staging the axilla. Ann Surg. 2011;254:243-51.

7. Nakamura R, Yamamoto N, Miyaki T, Itami M, Shina N, Ohtsuka M. Impact of sentinel lymph node biopsy by ultrasound-guided core needle biopsy for patients with suspicious node positive breast cancer. Breast Cancer. 2018;25:86-93.

8. Lloyd P, Theophilidou E, Newcombe RG, Pugh L, Goyal A. Axillary tumour burden in women with a fine-needle aspiration/core biopsy-proven positive node on ultrasonography compared to women with a positive sentinel node. Br J Surg. 2017;104:1811-5.

9. Curigliano G, Burstein HJ, E PW, Gnant M, Dubsky P, Loibl S, Colleoni M, Regan MM, Piccart-Gebhart M, Senn HJ, et al. De-escalating and escalating treatments for early-stage breast cancer: the St. Gallen International Expert Consensus Conference on the Primary Therapy of Early Breast Cancer 2017. Ann Oncol. 2017;28:1700-12.

10. Cameron D, Piccart-Gebhart MJ, Gelber RD, Procter M, Goldhirsch A, de Azambuja E, Castro G Jr, Untch M, Smith I, Gianni L, et al. 11 years' followup of trastuzumab after adjuvant chemotherapy in HER2-positive early breast cancer: final analysis of the HERceptin Adjuvant (HERA) trial. Lancet. 2017;389:1195-205.

11. Early Breast Cancer Trialists' Collaborative G. Aromatase inhibitors versus tamoxifen in early breast cancer: patient-level meta-analysis of the randomised trials. Lancet. 2015;386:1341-52.

12. Wolff AC, Hammond ME, Hicks DG, Dowsett M, McShane LM, Allison KH, Allred DC, Bartlett JM, Bilous M, Fitzgibbons P, et al. Recommendations for human epidermal growth factor receptor 2 testing in breast cancer: American Society of Clinical Oncology/College of American Pathologists clinical practice guideline update. J Clin Oncol. 2013;31:3997-4013.

13. Shigematsu H, Ozaki S, Yasui D, Zaitsu J, Taniyama D, Saitou A, Kuraoka K, Yamashiro H, Taniyama K. Comparison of CK-IHC assay on serial frozen sections, the OSNA assay, and in combination for intraoperative evaluation of SLN metastases in breast cancer. Breast Cancer. 2018;25:191-7.

14. Siegel RL, Miller KD, Jemal A. Cancer statistics, 2017. CA Cancer J Clin. 2017:67:7-30.

15. Carter $\mathrm{CL}$, Allen $\mathrm{C}$, Henson DE. Relation of tumor size, lymph node status, and survival in 24,740 breast cancer cases. Cancer. 1989;63:181-7.

16. Andersson $Y$, Frisell J, Sylvan M, de Boniface J, Bergkvist L. Breast cancer survival in relation to the metastatic tumor burden in axillary lymph nodes. J Clin Oncol. 2010;28:2868-73.

17. Andersson $Y$, Bergkvist L, Frisell J, de Boniface J. Long-term breast cancer survival in relation to the metastatic tumor burden in axillary lymph nodes. Breast Cancer Res Treat. 2018 Sep;171(2):359-369. https://doi.org/10.1007/ s10549-018-4820-0. Epub 2018 May 30. PMIS;29846847

18. Weaver DL, Ashikaga T, Krag DN, Skelly JM, Anderson SJ, Harlow SP, Julian TB, Mamounas EP, Wolmark N. Effect of occult metastases on survival in node-negative breast cancer. N Engl J Med. 2011;364:412-21.

19. Mittendorf EA, Ballman KV, McCall LM, Yi M, Sahin AA, Bedrosian I, Hansen N, Gabram S, Hurd T, Giuliano AE, Hunt KK. Evaluation of the stage IB 
designation of the American Joint Committee on Cancer staging system in breast cancer. J Clin Oncol. 2015;33:1119-27.

20. Goldhirsch A, Glick JH, Gelber RD, Coates AS, Thurlimann B, Senn HJ, Panel M. Meeting highlights: international expert consensus on the primary therapy of early breast cancer 2005. Ann Oncol. 2005;16:1569-83.

21. Cardoso F, van't Veer $\sqcup$, Bogaerts J, Slaets L, Viale G, Delaloge S, Pierga JY, Brain E, Causeret S, DeLorenzi M, et al. 70-gene signature as an aid to treatment decisions in early-stage breast cancer. N Engl J Med. 2016;375:717-29.

22. Tolaney SM, Barry WT, Dang CT, Yardley DA, Moy B, Marcom PK, Albain KS, Rugo HS, Ellis M, Shapira I, et al. Adjuvant paclitaxel and trastuzumab for node-negative, HER2-positive breast cancer. N Engl J Med. 2015;372:134-41.

23. Sanghani M, Balk EM, Cady B. Impact of axillary lymph node dissection on breast cancer outcome in clinically node negative patients: a systematic review and meta-analysis. Cancer. 2009;115:1613-20.

24. Gentilini O, Veronesi U. Abandoning sentinel lymph node biopsy in early breast cancer? A new trial in progress at the European Institute of Oncology of Milan (SOUND: Sentinel node vs Observation after axillary UltraSouND). Breast. 2012;21:678-81.

Ready to submit your research? Choose BMC and benefit from:

- fast, convenient online submission

- thorough peer review by experienced researchers in your field

- rapid publication on acceptance

- support for research data, including large and complex data types

- gold Open Access which fosters wider collaboration and increased citations

- maximum visibility for your research: over $100 \mathrm{M}$ website views per year

At BMC, research is always in progress.

Learn more biomedcentral.com/submissions 